\title{
Identification of candidate predictive and surrogate molecular markers for dasatinib in prostate cancer: rationale for patient selection and efficacy monitoring Xi-De Wang, Karen Reeves, Feng R Luo, Li-An Xu, Francis Lee, Edwin Clark and Fei Huang
}

Address: Pharmaceutical Research Institute, Bristol-Myers Squibb, Princeton, New Jersey, o8543, USA.

Correspondence: Xi-De Wang. Email: xi-de.wang@bms.com, Fei Huang. Email: fei.huang@bms.com

Published: 29 November 2007

Genome Biology 2007, 8:R255 (doi:10.1 I86/gb-2007-8-II-r255)

The electronic version of this article is the complete one and can be found online at http://genomebiology.com/2007/8/I I/R255
Received: 15 June 2007

Revised: 22 October 2007

Accepted: 29 November 2007

(C) 2007 Wang et al.; licensee BioMed Central Ltd.

This is an open access article distributed under the terms of the Creative Commons Attribution License (http://creativecommons.org/licenses/by/2.0), which permits unrestricted use, distribution, and reproduction in any medium, provided the original work is properly cited.

\begin{abstract}
Background: Dasatinib is a potent, multi-targeted kinase inhibitor that was recently approved for treatment of chronic myelogenous leukemia resistant to imatinib. To aid the clinical development of dasatinib in prostate cancer, we utilized preclinical models to identify potential molecular markers for patient stratification and efficacy monitoring.

Results: Using gene expression profiling, we first identified 174 genes whose expression was highly correlated with in vitro sensitivity of 16 cell lines and, thus, considered as candidate efficacy predictive markers. Among these are important prostatic cell lineage markers, cytokeratin 5, androgen receptor and prostate specific antigen. Our results indicate that 'basal type' cell lines with high expression of cytokeratin 5 and low expression of androgen receptor or prostate specific antigen are sensitive to dasatinib. To identify markers as surrogates for biological activity, we treated cell lines with dasatinib and identified genes whose expression was significantly modulated by the drug. Ten genes, including that encoding urokinase-type plasminogen activator (uPA), were found to not only be potential efficacy markers but also to have reduced expression upon dasatinib treatment. The down-regulation of uPA by dasatinib was drug-specific and correlated with the sensitivity of cell lines to dasatinib. Furthermore, EphA2, a target of dasatinib, was found to be a sensitivity biomarker.
\end{abstract}

Conclusion: Using the gene expression profiling approach and preclinical models, we have identified prostatic biomarkers that are associated with sensitivity to dasatinib. This study has provided a basis for clinical evaluation of a potential dasatinib efficacy signature in prostate cancer.

\section{Background}

Prostate cancer is the most common type of cancer in men of western countries. It is estimated that each year about 230,000 men in the United States alone are diagnosed with prostate cancer and approximately 30,00o die of this disease [1]. Although targeted therapeutics have shown promise for cancer patients, their use in treating prostate cancer is still limited. Current regimens available for prostate cancer 
patients include conventional surgery, radiation and hormonal therapies for early stage tumors, and taxane-based chemotherapy for late stage metastatic tumors [2,3]. There is a clear unmet medical need to develop targeted therapeutics for prostate cancer.

Biomarkers can dictate the successful clinical development of novel anti-cancer drugs and the clinical benefits that patients can derive from -targeted therapeutics. Using expression of HER2 as a patient selection criterion has allowed the successful development of trastuzumab, a monoclonal antibody therapy targeting HER2 in breast cancer. Breast cancer patients identified to over-express HER2 who subsequently receive this therapy show a significant response rate and profound clinical benefits [4]. In contrast, failure to identify and use robust biomarkers in trials for innovative medicines can result in failed approval and/or dramatically delayed clinical development [5,6]. Such examples highlight the need in clinical development to identify molecular biomarkers that will guide patient selection and enable monitoring of drug efficacy at the molecular level.

Dasatinib is a potent, orally available small molecule inhibitor that targets multiple cytosolic or membrane-bound tyrosine kinases, including Src-family kinases (SFKs), Bcr-Abl, c-kit, platelet-derived growth factor receptor (PDGFR) $\beta$ and EphA2 $[7,8]$. Due to its potency against leukemic cancer cell lines harboring $B C R-A B L$ mutations [9], the clear and imminent need for overcoming imatinib resistance, and the profound clinical benefit demonstrated in clinical trials, dasatinib was recently approved for use in chronic myelogenous leukemia and Philadelphia chromosome-positive acute lymphoblastic leukemia that are resistant or intolerant to imatinib [10]. In contrast, other targets of dasatinib (for example, SFKs, EphA2) have yet to be clinically validated. The involvement of SFKs in a number of cellular processes, such as cell migration, adhesion and angiogenesis, as well as participation of SFKs in a number of clinically relevant pathways (for example, the epidermal growth factor receptor (EGFR) pathway) $[11,12]$ have prompted investigations into the potential use of dasatinib in solid tumors [13]. Such investigations would, as discussed above, best be supported by the use of molecular biomarkers.

To support the development of dasatinib for use in prostate cancer we employed prostate cancer cell lines as preclinical models to identify molecular biomarkers whose expression correlated with the sensitivity to dasatinib and could potentially be used as surrogates to monitor the biological effects of dasatinib in patients. First, we identified candidate predictor genes with baseline expression levels correlated with sensitivity to dasatinib. Next we identified genes that were significantly modulated by dasatinib treatment. Urokinase-type plasminogen activator $(u P A)$ was observed to be on both lists, suggesting it may be a candidate predictive and 'surrogate' biomarker. Additionally, EphA2, a target of dasatinib, is a candidate predictor of efficacy in both prostate and breast cancer. Finally, the observed sensitivity to dasatinib of prostate cancer cell lines with expression of basal cell markers, together with a similar observation in breast cancer cell lines [8], suggests a common mechanism of sensitivity to SFKs and a role of SFKs in epithelial tumors derived from the basal layer.

\section{Results \\ Identification of markers correlated with dasatinib sensitivity}

The aim of this study was to identify both predictive and surrogate biomarkers that could potentially assist the clinical development of dasatinib in prostate cancer. As outlined in Figure 1a, our strategy was first to identify genes whose baseline expression levels correlated with drug sensitivity and passed additional variation requirements to obtain a candidate predictive marker list. Then genes whose expression was modulated by dasatinib in a drug treatment study were identified and compared to the candidate predictive biomarker list to identify genes that were not only correlated with drug sensitivity but also modulated by drug treatment.

The half maximal inhibitory concentration $\left(\mathrm{IC}_{50}\right)$ values of 16 prostate cancer cell lines to dasatinib were determined as shown in Figure $1 \mathrm{~b}$. Based on the $\mathrm{IC}_{50}$ values, cell lines were classified into two groups: 11 cell lines with $\mathrm{IC}_{50}$ values lower than $200 \mathrm{nM}$ (within the range of dasatinib plasma concentrations clinically achieved) were designated as sensitive; and 5 cell lines with $\mathrm{IC}_{50}$ values greater than or equal to $2 \mu \mathrm{M}$ (greater than the highest drug concentration clinically achieved in plasma) were considered resistant. It is noted that the sensitivity or resistance of cell lines is not correlated with their doubling times, as both the sensitive and resistant groups consist of cell lines that grow fast or relatively slowly.

Baseline gene expression profiling of the 16 cell lines was performed using Affymetrix gene chips. Two statistical tests (one-way ANOVA and correlation to $\log _{2} \mathrm{IC}_{50}$ values) were performed to identify genes that were differentially expressed between sensitive and resistant groups $(5,961$ probe sets with $p<0.05$ ) and those that were highly correlated with $\mathrm{IC}_{50}$ (4,575 probe sets with $p<0.05)$, respectively; 4,248 probe sets overlapped in these two analyses, suggesting that the categorization of sensitive and resistant groups reflected well the sensitivity of the cells $\left(\mathrm{IC}_{50}\right)$ to dasatinib. The list was further filtered with a requirement for a $10 \%$ coefficient of variation (CV) across all samples and a minimum 3-fold differential expression between the sensitive and resistant groups, resulting in the selection of 213 probe sets. Expressed sequence tags (ESTs) and duplicate probe sets were further removed to generate the candidate predictive marker list of 174 genes (Additional data file 1 ). 


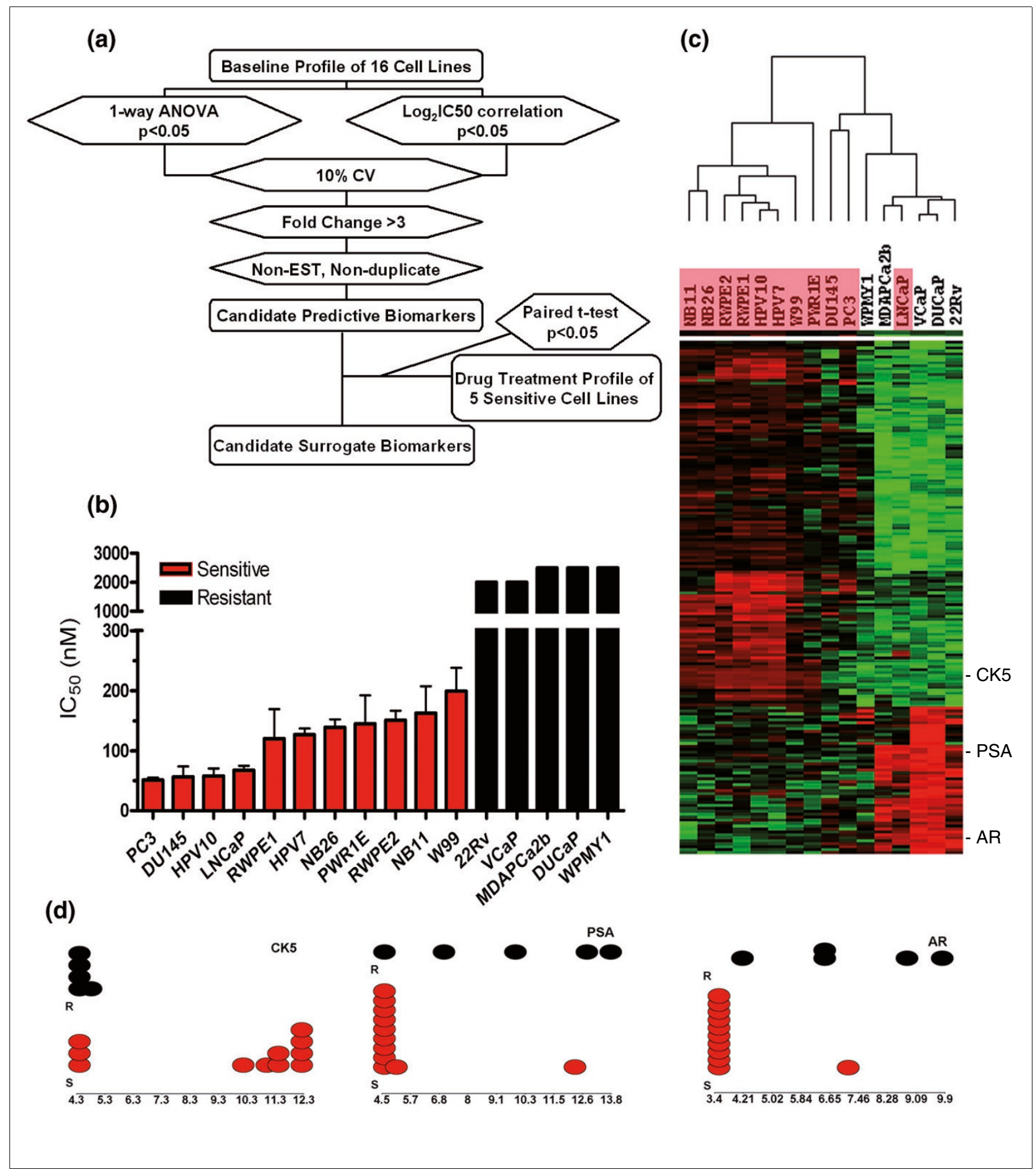

\section{Figure I}

Identification of biomarkers correlated with sensitivity to dasatinib. (a) Discovery strategy to identify potential predictive and surrogate biomarkers. (b) $\mathrm{IC}_{50}$ determination and sensitivity classification of 16 prostate cancer cell lines to dasatinib. (c) Cluster analysis showing the relative expression pattern of 174 genes that were highly correlated with dasatinib sensitivity/resistance classification of 16 cell lines. The dasatinib-sensitive cell lines are highlighted in red, and the position of three important prostatic cell markers, CK5, PSA and AR, are marked on the heatmap. These genes have differential expression of more than three-fold between the sensitive and resistant groups. (d) Relative baseline gene expression of CK5, PSA and AR in the 16 cell lines. The resistant cells are in black and the sensitive cells are in red. The values on the $\mathrm{x}$-axes are expression level in $\log _{2}$-scale. 
The expression pattern of these 174 genes on the 16 cell lines was visualized by cluster analysis. As shown in Figure 1c, these 16 cell lines were separated into two major groups (the dasatinib-sensitive cell lines are marked in red). Interestingly, the cell lines in the left cluster were all sensitive to dasatinib; within the right cluster, DU145, PC3 and LNCaP cells were also highly sensitive to dasatinib.

Genes of biological interest in Additional data file 1 include EGFR pathway genes such as amphiregulin and epiregulin, transforming growth factor pathway genes such as $T G F \alpha$, $T G F \beta 2$ and TGF $\beta R I I$, as well as other receptor tyrosine kinases, such as the Met proto-oncogene and fibroblast growth factor receptor 2. These genes were more highly expressed in sensitive cell lines. Most strikingly, several important prostatic cell markers, such prostate specific antigen (PSA; also known as kallikrein 3) and androgen receptor (AR) were over-expressed in the resistant cell lines, while cytokeratin (CK) 5 was highly expressed in the sensitive cell lines (Figure 1c).

The relative expression levels of $C_{5}, P S A$ and $A R$ in these 16 cell lines are shown in more detail in Figure 1d. We observed that resistant cell lines all express very low levels of $C_{5}$ and sensitive cell lines all express high levels of $C_{5}$, except for DU145, PC3 and LNCaP cells (Figure 1c). As CK5 is a basal cell marker for the prostatic cell lineage, these data suggest that cells exhibiting the basal phenotype are sensitive to dasatinib and that cells expressing lower levels of $\mathrm{CK}_{5}$ tend to be resistant. The expression pattern of PSA and AR, two luminal cell markers, complementarily reinforces the above observation. While higher expression of $P S A$ and $A R$ is correlated with drug resistance, lower expression of these two genes is correlated with dasatinib sensitivity. The LNCaP cell line, which was sensitive to dasatinib and expressed high levels of PSA and $A R$, is the only exception to this observation out of five cell lines (MDAPCa2b, LNCaP, VCaP, DUCaP, and 22Rv) found to express higher levels of $P S A$ and $A R$ (Figure 1c).

\section{Identification of markers that are also modulated by dasatinib treatment}

Five dasatinib-sensitive cell lines, including two $\mathrm{CK}_{5}{ }^{-}$ expressing (PWR1E and RWPE2) and three $C K_{5}$-nonexpressing cell lines (PC3, DU145 and LNCaP), were treated with dasatinib. Comparison by paired $t$-test of gene expression profiles between post-dasatinib treatment and mock treatment for each cell line revealed that 1,628 probe sets were significantly modulated by drug treatment ( $p<0.05)$. Comparison of these 1,628 probe sets with the list of candidate predictive markers (Additional data file 1) indicated that 10 genes were common to both lists. These ten genes, which may be potentially used to predict sensitivity to dasatinib and to serve as surrogates for drug activity, are indicated in the last column of Additional data file 1. Interestingly, all ten of these genes were highly expressed in sensitive cell lines and decreased in expression after dasatinib treatment. These

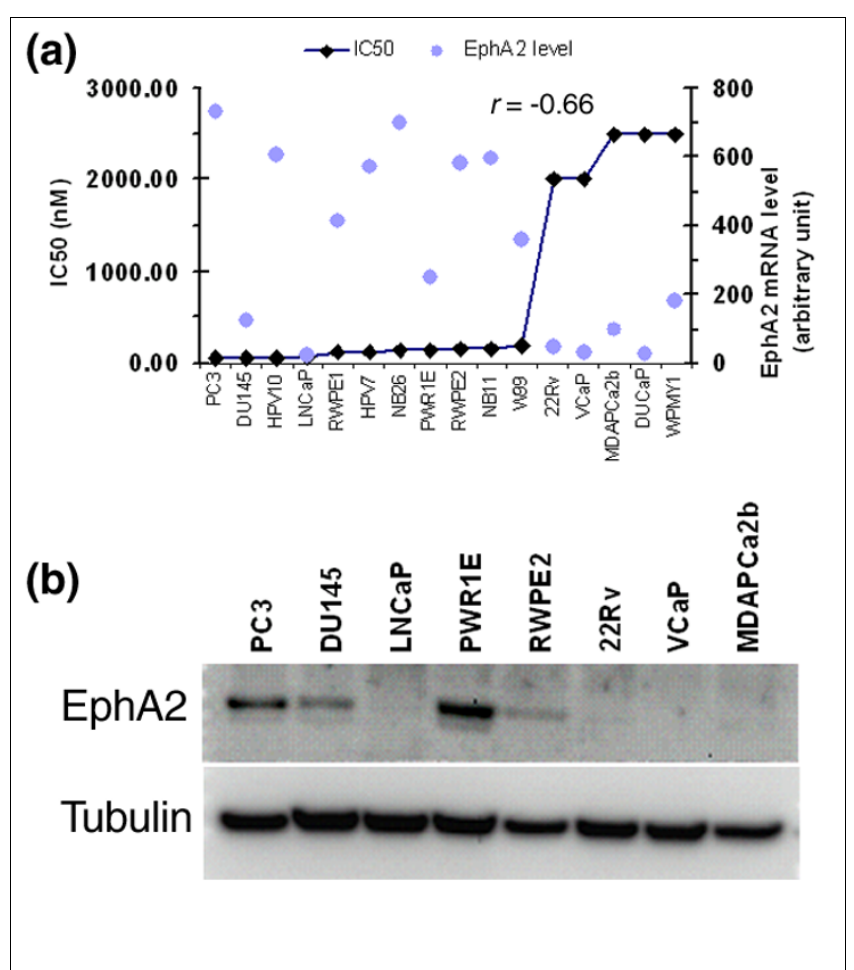

Figure 2

Correlation of EphA2 gene expression with sensitivity to dasatinib. (a) Negative correlation between the expression levels of EphA2 (black diamonds) and the $\mathrm{IC}_{50}$ (gray circles) values for the 16 cell lines. The coefficient of the Pearson correlation is -0.66 , indicating a high reverse correlation. (b) Expression of EphA2 protein in five sensitive and three resistant cell lines. Overall, the protein expression levels in these cell lines correlated well with the mRNA levels detected by microarray analysis.

genes include those encoding epiregulin, a component in the EGFR pathway, FHL2 and AXL kinases, and uPA. Three of these ten genes including $L A M C 2, E R E G$ and $U P A$ encode proteins that are secreted to the extracellular matrix.. This set of genes may represent genes whose expression are under the regulation of genes targeted by dasatinib.

\section{Common biomarkers identified in prostate and breast preclinical studies}

To facilitate clinical development of dasatinib for breast cancer, a similar preclinical biomarker study was performed in this laboratory [8]. Biomarkers predictive of dasatinib sensitivity in breast cancer cell lines were identified and are currently being assessed in clinical trials. Since the majority of breast tumors are also epithelial in origin, we compared the biomarkers discovered in the current prostate cancer study with those identified in the breast cancer study. To this end, in addition to the 174 genes noted above, we also included probe sets in the list of 1,475 probe sets (after the 10\% CV step, but before the fold-change $>3$ filter step) that were also significantly modulated by dasatinib (that is, present in the list of 1,628 probes) as candidate prostate biomarkers and compared them to the breast cancer biomarker list of 161 genes 
[8]. Fourteen genes were identified as common biomarkers in both tissue types (Additional data file 2). Notably, EphA2, a dasatinib target, was significantly correlated with dasatinib sensitivity in both prostate and breast cancer cell lines. As shown in Additional data file 2, the mean expression of EphA2 was significantly higher in sensitive cell lines than in resistant cell lines (2.69-fold with $p=0.005$ by $t$-test).

As shown in Figure 2a, the expression level of EphA2, as detected by microarray baseline profiling, was correlated with the $\mathrm{IC}_{50}$ values of the prostate cell lines (higher EphA2 expression with lower $\mathrm{IC}_{50}$ or high sensitivity, Pearson correlation coefficient $=-0.66$ ). As a validation, we also performed western blot analysis to examine the expression of EphA2 protein in five sensitive and three resistant cell lines (Figure 2b). Overall, those cell lines with higher levels of EphA2 mRNA expressed relatively higher levels of EphA2 protein, indicating good concordance between gene and protein expression for EphA2. While the EphA2 RNA level in DU145 cells was relatively low compared to the other sensitive cell lines, its protein level appeared comparable, suggesting that EphA2 expression is also regulated at the protein translation or stabilization levels. Our western blot results on EphA2 protein in $\mathrm{PC}_{3}$, DU145 and LNCaP cells are consistent with a previous report [14]. With the correlation of its expression with dasatinib sensitivity in cell lines, and being a target of the drug, EphA2 appears to be a strong candidate biomarker for dasatinib in prostate cancer.

\section{Down-regulation of uPA expression by dasatinib}

Since the secreted protein uPA is regulated by SFKs [15], we further evaluated the expression of the $u P A$ gene and its modulation by dasatinib. As shown in Figure 3a (and also Additional data file 1), the expression level of the $u P A$ gene in sensitive cell lines was significantly higher than in resistant cell lines. A second probe set for the $u P A$ gene also showed a similar expression pattern. Additionally, three probe sets for the uPA receptor, which partners with uPA in its function, all showed a similar expression pattern as $u P A$ in these cell lines (data not shown).

The down-regulation of $u P A$ mRNA expression upon dasatinib treatment was observed (Figure $3 \mathrm{~b}$ ). A relatively mild reduction of $u P A$ expression was observed in two $\mathrm{CK}_{5}-$ expressing cells (PWR1E and RWPE2) while the reduction in $\mathrm{PC}_{3}$ and DU145 cells was much stronger (approximately $50 \%)$. LNCaP cells, which express a much lower level of $u P A$, also showed a reduction upon dasatinib treatment. When we extended the same drug treatment study in three resistant cell lines, 22Rv, VCaP and MDAPCa2b (Figure 3c), the magnitude of $u P A$ reduction by dasatinib was correlated nicely with the sensitivity of cells to dasatinib $(r=0.72)$, with the highest reduction seen in the most sensitive cell line. This suggests uPA is a potential surrogate biomarker for the biological effect of dasatinib. Furthermore, in a multiple-dose treatment study with $\mathrm{PC}_{3}$ cells, we found that the reduction of $u P A$
mRNA level by dasatinib at $4 \mathrm{~h}$ was minimal for all doses compared to untreated control, but the changes were dramatic at $24 \mathrm{~h}$ and occurred in a dose-dependent fashion (Figure $3 \mathrm{~d})$.

The down-regulation of uPA expression by dasatinib was also seen at the protein level. Using an enzyme-linked immunosorbent assay (ELISA), we found in a time course experiment that the amount of uPA protein secreted by $\mathrm{PC}_{3}$ cells into the growth medium after $24 \mathrm{~h}$ was reduced by dasatinib treatment, and the extent of this reduction was dose-dependent, as shown in Figure 3e. As a control, when using a cytotoxic agent, paclitaxel, we did not see a dose-dependent reduction in the secreted uPA protein level, suggesting that down-regulation of uPA expression is not a consequence of cell growth inhibition.

\section{Rationale for patient stratification in dasatinib prostate cancer trials}

Based on their differential expression, we reasoned that $\mathrm{CK}_{5}$, PSA, and AR could serve as predictive biomarkers for identification of subtypes of prostate tumors that would benefit from dasatinib treatment. We also reasoned that uPA and EphA2 could potentially be used as markers to monitor dasatinib activity because of their correlation with drug sensitivity and the links with dasatinib's mechanisms of action. The expression patterns of these 5 genes in the 16 cell lines are shown in Figure 4a. Five dasatinib resistant cell lines, WPMY1, MDAPCa2b, 22Rv, VCaP, and DUCaP, all expressed high levels of $A R$ and $P S A$ and low levels of $C_{5}, u P A$ and $E p h A 2$. In contrast, sensitive cell lines expressed low levels of $A R$ and $P S A$, with the exception of $\mathrm{LNCaP}$, and high levels of $u P A, E p h A 2$ and/or $C K 5$.

The dynamic range in the expression of these 5 genes and the approximate patient population exhibiting dasatinib-responsive expression patterns were examined using a previously published prostate tumor data set consisting of 52 tumor samples [16]. As shown in Figure 4b, nearly 44\% (23/52, sample ID labeled in red) of the prostate tumors showed the 'dasatinib-responsive' expression patterns (that is, low $A R$ and $P S A$ and high $u P A, E p h A 2$ and/or $C K 5$ ). In the remaining approximately $56 \%$ of tumors, the expression of $A R$ and $P S A$ were concordantly relatively high and the expression of $u P A$ and EphA2 were relatively low. There were certain degrees of co-expression as well as mutually exclusive expression of $A R$ and $C_{5}$ in this data set, reminiscent of the expression pattern of these two genes in basal, intermediate and luminal cells of normal prostatic epithelium $[17,18]$.

\section{Discussion}

The ideal scenario for identifying biomarkers for clinical use is to use samples obtained from patients undergoing therapy with the investigational drug and to analyze gene expression data in the context of patient response data. Since dasatinib is 




Figure 3

\begin{abstract}
Figure 3
uPA gene expression and regulation by dasatinib analyzed at mRNA and protein levels. (a) Differential baseline expression of uPA gene between resistant $(R$, in black) and sensitive $(S$, in red) cell lines. The $x$-axis values are the expression level in $\log _{2}$-scale. The resistant cell line expressing a high level of uPA was WPMYI and the sensitive cell line expressing a low level of UPA was LNCaP. (b) Down-regulation of UPA mRNA level by dasatinib treatment in five sensitive cell lines. The cells were treated with $100 \mathrm{nM}$ dasatinib (+D) or DMSO (Ctrl) for $48 \mathrm{~h}$. The $p$ value was 0.048 by paired $t$-test, indicating a significant reduction of $u P A$ mRNA following dasatinib treatment. (c) Correlation between dasatinib-induced $u P A$ mRNA down-regulation with the sensitivity of cell lines to dasatinib. In addition to the five sensitive cell lines, three dasatinib-resistant cell lines, $22 \mathrm{Rv}$, MDAPCa2b, and VCaP, were also treated with dasatinib as in (b). The extent of $u P A$ down-regulation by dasatinib ( $y$-axis) was negatively correlated with the $\log _{2} \mid C_{50}$ values ( $x$-axis) of these eight cell lines. (d) Dose-dependent down-regulation of uPA mRNA expression in PC3 cells. Cells were treated with or without different concentrations of dasatinib for 4 or $24 \mathrm{~h}$. The uPA expression level relative to control is shown on the $y$-axis. (e) Dose-dependent inhibition of secreted uPA protein in PC 3 cells by dasatinib but not by paclitaxel. Cells were treated with different doses of dasatinib, paclitaxel or DMSO for $24 \mathrm{~h}$. The amount of uPA protein secreted into the culture medium by 50,000 viable cells was assessed by ELISA assay.
\end{abstract}

a novel agent in early clinical development, using preclinical models to identify candidate biomarkers for assisting clinical development appears the best option. In this study, we used 16 prostate cell lines to identify biomarkers that were correlated with the sensitivity of cells and with the mechanisms of drug action. These biomarkers could potentially be used for predicting and monitoring dasatinib response. In particular, we identified five genes $\left(A R, P S A, C_{5}, u P A\right.$ and $E p h A 2)$ that were highly associated with drug sensitivity/ resistance and/or modulated by drug treatment. Consistent with our observation in breast cancer cell lines [8], it appears that basal-type prostate cancer cells expressing low levels of $A R$ and PSA and a high level of $C_{5}$ are most responsive to dasatinib treatment. Higher expression levels of $u P A$ and $E p h A 2$ may also help to define patients that will benefit from dasatinib treatment. In addition, $u P A$ expression was regulated by dasatinib and such regulation was highly correlated with the sensitivity of cells to dasatinib, suggesting that $u P A$ expression can potentially be used as a surrogate marker to monitor dasatinib activity. As a potent inhibitor against Abl, c-kit, PDGFR $\beta$ and, in particular, Src and EphA2, which have been shown to play important roles in prostatic tumorigenesis $[13,14]$, dasatinib holds high promise as a potential treatment for prostate cancer. It is noted that imatinib, which inhibits three of these targets, including Abl, c-kit and PDGFR kinases, showed minimal efficacy in early clinical testing with a small number of patients [19,20]. Identification of these preclinical candidate markers and further validation of them in early clinical trials would facilitate patient stratification in registration trials of dasatinib.

In our data analysis, we used an approach that emphasized both statistical significance and high-fold differential gene expression between subgroups. Since we have only 16 cell 


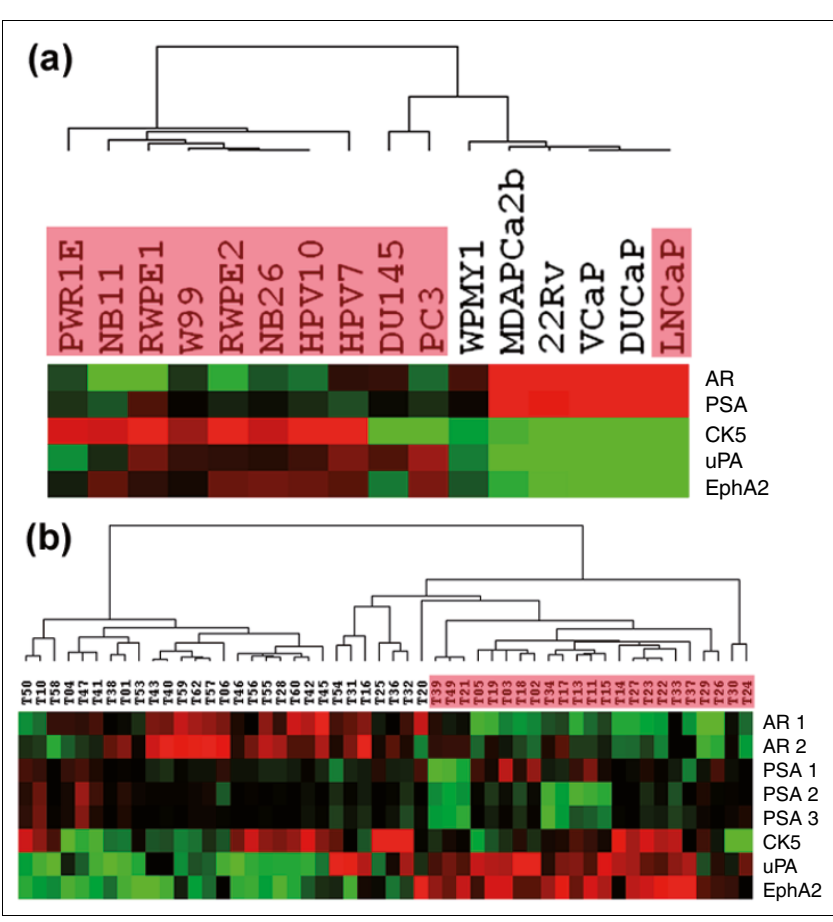

\section{Figure 4}

Expression pattern of a five-gene model in prostate cell lines and tumors. (a) Hierarchical clustering of five genes, AR, PSA, CK5, uPA, and EphA2, in prostate cell lines. AR and PSA are two luminal cell markers, and CK5 is a basal cell marker. The resistant cell lines were separated from the majority of the sensitive cells (labeled in red) by these five genes. Note the high expression of CK5 and/or UPA, and EphA2 in sensitive cells and the nearly opposite expression pattern in resistant cells. (b) The expression pattern of these 5 genes in 52 prostate tumors. The gene expression of this published data set was profiled on Affymetrix HG-U95 gene chips. Two $A R$, three PSA probe sets and one CK5, uPA and EphA2 probe set are available on the chip and were retrieved for cluster analysis. The tumor samples exhibiting a dasatinib-sensitive pattern, that is, with low $A R$ and PSA expression and high CK5 and/or UPA, and EphA2 expression are highlighted in red.

lines, a pool that may not be necessarily large enough for stringent statistical analyses, we included other requirements, including stringent variation and fold change filters. Notably, the approach we undertook was essentially consistent with that reported by a recent publication from the MicroArray Quality Control initiative led by the Food and Drug Administration, which showed that gene lists ranked by fold change and filtered with non-stringent yet statistically significant tests were more reproducible across platforms than lists generated with other analytical strategies [21]. The result we obtained is biologically meaningful as we identified subtypes of cells sensitive to dasatinib that reflect normal and/or pathogenic prostatic biology and genes that reflect the function of dasatinib targets. In addition, a number of biomarkers identified were also observed from a recently published breast cancer study [8].

Inside normal prostatic epithelium, there exist two major types of epithelial cells, basal and luminal epithelial cells. Sev- eral recent studies suggest that there may be further divisions of epithelial cells into subtypes that are more specialized in function. Among these subtypes are prostatic stem cells, transit amplifying cells, intermediate cells and secretary luminal cells, which can be differentiated based on their expression pattern of certain cellular markers. In our study, we found that cells with low $A R$ and $P S A$ expression and high $C_{5}$ expression represent a sub-population that is sensitive to dasatinib. This expression pattern matches that of epithelial cells residing in the basal compartment, which can potentially be prostatic stem cells or transit amplifying cells. Since these two types of cells are able to self-renew and quickly proliferate to give rise to new or more differentiated cells, an intrinsic relationship may exist between the expression and function of dasatinib targets such as the SFK LYN and the proliferation of these cells [11]. Indeed, LYN is more highly expressed in the basal layer than in the luminal layer, and in tumors LYN tends to be more highly expressed in less differentiated regions [22]. Our result that basal type prostatic cells are sensitive to dasatinib resonates with recent reports showing that dasatinib is active on a basal subtype of breast cancer cells [8,23]. Notably, gene expression profiling studies with a large set of breast tumors or cancer cell lines have identified basal epithelial types of tumors or cells that highly express $C_{55}, E G F R$ and SFK LYN [24,25], which suggests that basal epithelial cells, as well as SFK and their cooperating partners, such as EGFR, have important roles in breast tumorigenesis [12].

In contrast to the well established and validated classification of breast cancer subtypes that has been used in the clinic to aid patient stratification $[25,26]$, molecular classification of prostate cancer using genome-wide profiling techniques has been explored [27] but lacks validation with independent tumor cohorts. This may be a result of the heterogeneity of prostate tumors and the difficulty in obtaining biopsies from prostate cancer patients. It remains to be determined whether the molecular subtypes of prostate cancer identified through molecular profiling mimic the epithelial subtypes seen in normal prostatic epithelium, as is seen in breast cancer $[25,26]$. From our analysis of the data set published by Singh et al. [16], prostate cancer subtypes that express lower levels of $A R$ and PSA and higher levels of $C_{5}$ (that is, the basal type) and those with the opposite expression pattern (that is, the luminal type) apparently exist. The derivation of prostate cancer cell lines such as MDAPCa2b, VCaP, DUCaP and LNCaP also demonstrates the existence of a luminal type of prostate cancer. The low expression levels of $P S A / A R$ as well as $C_{5}$ in $\mathrm{PC}_{3}$ and DU145 cells may also suggest subtypes other than luminal and basal. It is noted that the subtype with high expression of $C_{5} 5$ and low expression of $A R$ and $P S A$ seen in our study is mainly based on immortalized prostatic cell lines. However, a recent study showed that sub-cell populations with varying degrees of differentiation co-exist in both $A R$ expressing LAPC-4/LAPC-9 human cancer xenograft models and $\mathrm{PC}_{3}$ and DU145 cancer cell populations. The sub-population that expressed CD44, another basal cell marker [28], 
showed the highest tumorigenicity [29]. These results strongly suggest that a subtype of cells with the phenotypes of normal basal cells exists in prostate tumors and may play a major role in determining tumor malignancy.

We found in our study that LNCaP cells, an androgen-sensitive prostate cancer cell line, exhibited sensitivity to dasatinib distinct from other androgen-sensitive cell lines, including 22Rv, MDAPCa2b, VCaP and DuCaP. Transcriptionally, these 5 cell lines resemble each other in terms of expression of $A R / P S A$, the 174 genes associated with in vitro response to dasatinib, as well as global gene expression (Figure 1c; other data not shown). It is not clear what mechanism in LNCaP cells caused dasatinib susceptibility. The $A R$ gene mutation may be an appealing, but not necessarily straight-forward, explanation as LNCaP cells possess one T877A mutation and other cells either have no mutation ( $\mathrm{VCaP}$ and $\mathrm{DuCaP}$ ) or have other mutations (22Rv, H874Y) or additional types of mutations (MDAPCa2b, L701H and T877A) [30]. Alteration of the expression or sequence of $A R$ may affect the function of $\mathrm{AR}$ in terms of binding to androgen [31] or cross-talk with growth factor and receptor pathways such as phosphorylation of AR by signaling cascades [32]. The inhibition of growth factor and receptor pathways by dasatinib may also induce the cells to re-establish the balance of signaling networks and modify the mode of action of AR. It is also possible that the function of one or more targets of dasatinib is indispensable for LNCaP cell growth.

By identifying genes whose expression was altered by drug treatment, we found a set of genes that may correlate with the mechanisms of action of dasatinib. This is desirable in drug development in two ways. First, markers correlated with mechanisms of action would enhance the level of confidence when biomarkers are used in clinical testing for the specific drug. Second, knowledge of drug efficacy through sensitive molecular testing can help to prevent premature discontinuation of clinical studies due to low clinical responses in early stage trials with small numbers of patients. In particular, while dasatinib is potent in inhibiting cell adhesion, migration and invasion, it appears in preclinical models to be cytostatic rather than cytotoxic. Thus, sensitive surrogate markers become critical for an evaluation of drug efficacy in early trials. In our study, we found that uPA, a downstream target of Src kinase, was modulated significantly by dasatinib, and such down-regulation was specifically caused by dasatinib and not by other cytotoxic agents, such as paclitaxel. In addition, the magnitude of drug-induced uPA reduction correlated very well with the sensitivity of cell lines to dasatinib, with higher reduction observed in sensitive cell lines and little or no change in resistant ones. These data suggest that uPA could potentially be used as a surrogate biomarker for monitoring the effect of dasatinib. More excitingly, uPA has been demonstrated to play an important role in prostatic tumorigenesis in numerous studies. For example, it is highly expressed in high grade prostate tumors and metastases [33], and when a tumor progresses to androgen independence, the level of uPA expression is enhanced [34]. In addition, RNA interference-induced knockdown of uPA inhibits invasion, survival and in vivo tumorigenicity of prostate cancer cells [35]. In the clinic, elevated uPA plasma level or uPA-uPA receptor densities are correlated with prostate cancer invasion, metastasis and poorer survival in prostate cancer $[36,37]$ and its value as a prognostic marker has been well established in breast cancer [38,39].

Among the candidate predictive biomarker genes, several are components of signaling pathways important for cell survival and proliferation, such as the EGF-EGFR, transforming growth factor (TGF)-TGF receptor, fibroblast growth factor receptor and Met pathways. As an intracellular tyrosine kinase, Src can act as a signal transducer downstream of these receptor tyrosine kinases $[11,12,40]$. Alternatively, Src kinase may function independently of one or more pathways. Although the mechanisms of either co-operation or cross-talk of these pathways with the Src-mediated pathway in prostate cancer is not quite clear, they may still represent candidate target pathways for combination therapies to achieve additive or synergistic effects. This observation may provide insight for future clinical development strategies.

\section{Conclusion}

Our study, utilizing a gene expression profiling approach in preclinical models, has identified prostatic biomarkers that are associated with sensitivity to dasatinib, a novel multi-targeted kinase inhibitor. In particular, five biomarkers (AR, PSA, CK5, uPA, and EphA2) could potentially help patient stratification and allow molecular monitoring of dasatinib activity in clinical trials. These markers are currently under early phase clinical evaluation using methods such as immunohistochemistry, ELISA or RT-PCR to identify potential associations with drug efficacy. In all, this preclinical study has provided a basis for clinical exploration, validation, and further implementation of a potential dasatinib efficacy signature for prostate cancer.

\section{Materials and methods \\ Cell lines and cell culture}

All cell lines were obtained from the American Type Culture Collection (Manassas, VA, USA), except DUCaP, which was obtained from Dr Kenneth Pienta at the University of Michigan. PWR1E and MDAPCa2b cells were grown in BRFF-HPC1 serum-free medium (AthenaES, Baltimore, MD, USA), and all other cells were cultured in RPMI 1640 supplemented with $10 \%$ fetal bovine serum, $100 \mathrm{IU} / \mathrm{ml}$ penicillin, and $100 \mathrm{mg} / \mathrm{ml}$ streptomycin (Invitrogen, Carlsbad, CA, USA). DUCaP cells were maintained in the presence of an immortalized mouse fibroblast cell line, which formed a layer beneath the DUCaP cells that easily dislodged. Cells were incubated at $37^{\circ} \mathrm{C}$ with circulation of $5 \% \mathrm{CO}_{2}$. 


\section{In vitro cell proliferation assay}

The effect of dasatinib treatment on cell proliferation was measured using a tetrazolium compound-based colorimetric method (MTS kit, Promega, Madison, WI, USA). The optimum number of cells to seed in 96-well plates to achieve linearity was determined in pilot experiments. Cells were plated at a density of 2,000-5,000 cells/well into 96-well plates and cultured overnight. Cells were then treated with dasatinib at serially diluted concentrations. Three days later, the reagent solution was added to the medium and the absorbance was measured on a SpectraMax photometric plate reader (Molecular Devices, Sunnyvale, CA, USA) at $490 \mathrm{~nm}$. The results were plotted against drug concentrations and $\mathrm{IC}_{50}$ values were calculated using Prism4 software (GraphPad, San Diego, CA, USA). The $\mathrm{IC}_{50}$ was the concentration of dasatinib that would reduce cell proliferation by $50 \%$ compared to control. A minimum of three independent assays were performed for each cell line. The mean \pm standard deviation was calculated except for cell lines that were highly resistant to the compound, for which accurate $\mathrm{IC}_{50}$ values were hard to obtain. In the latter case, the concentration of dasatinib that was able to consistently reduce cell proliferation was used as the $\mathrm{IC}_{50}$. The inhibition of dasatinib on cell growth was also visually confirmed under the microscope. Dasatinib stock, dissolved in DMSO, was $10 \mathrm{mg} / \mathrm{ml}$.

\section{Microarray analysis}

Affymetrix HG-U133A 2.0 gene chips containing approximately 22,000 probe sets were used for gene expression profiling (Affymetrix, Santa Clara, CA, USA). Total RNA was isolated from the cells using the RNeasy kits (Qiagen, Valencia, CA, USA). The quality of the RNA was assessed using an Agilent 2100 Bioanalyzer (Agilent, Santa Clara, CA, USA). Total RNA (10 $\mu \mathrm{g}$ ) was used for the preparation of biotinlabeled cRNA. Chip hybridization, scanning and data acquisition were performed according to the Expression Analysis Technical Manual provided by the manufacturer.

\section{Data analysis}

The raw expression data were normalized using an RMA algorithm and analyzed in Partek Discovery Suite software (Partek, St Louis, MO, USA). Two statistical analyses, including one-way ANOVA (for comparison of gene expression between sensitive and resistant cell line groups) and Pearson correlation (between gene expression level and $\log _{2} \mathrm{IC}_{50}$ values) were performed to identify genes whose baseline expression levels correlated with sensitivity to dasatinib in 16 prostate cell lines (a $p$ value $<0.05$ in both analyses was required for inclusion). The gene list was further narrowed down by variation filters (10\% CV of gene expression values across all samples and a minimum 3-fold differential expression between sensitive and resistant cell groups as defined by $\mathrm{IC}_{5 \mathrm{O}}$ ). ESTs and gene duplicates were eliminated from the final list. Gene expression profiles of drug treated (100 $\mathrm{nM}$ for 2 days) cell lines were compared with those of DMSO control using paired $t$-test ( $p$ value $<0.05$ ). Clustering analysis was performed using GeneCluster software and heatmaps were generated with red and green indicating high or low expression, respectively [41]. Gene expression raw data have been deposited in the Gene Expression Omnibus database (accession number GSE9633).

\section{Western blot analysis}

Cell lysates were prepared from asynchronously growing cells using the RIPA buffer supplemented with protease (Roche Diagnostics, Indianapolis, IN, USA) and phosphatase inhibitor (Sigma, St Louis, MO, USA) cocktails. Protein concentration was determined using the BCA kit (Pierce, Rockford, IL, USA). Lystate (30 $\mu \mathrm{g})$ was loaded and resolved on NuPAGE Novex 4-12\% Bis-Tris gel (Invitrogen, Carlsbad, CA, USA). The blots were probed with mouse monoclonal anti-EphA2 (Upstate Biotechnology, Lake Placid, NY, USA) and antitubulin antibodies (Abcam, Cambridge, MA, USA) and developed with chemiluminescence reagent ECL Plus (GE Healthcare, Piscataway, NJ, USA).

\section{uPA protein ELISA assay}

Cells were seeded in 24-well plates at a density of 25,00o cells per well. Two days later, the cells were washed twice with phosphate-buffered saline and the medium was changed to RPMI 1640 containing $0.1 \%$ fetal bovine serum and different concentrations of dasatinib or paclitaxel. Medium was sampled at $0,2,4,8,24$, and $48 \mathrm{~h}$ and immediately centrifuged at $10,000 \mathrm{~g}$ for 5 minutes. The supernatants were frozen at $-80^{\circ} \mathrm{C}$ until analysis. The total number of cells was quantified using a cell counter, and the number of viable cells was assessed with Trypan Blue. The amount of uPA protein in the supernatant was determined using the uPA ELISA kit (America Diagnostica, Stamford, CT, USA), and the concentrations of uPA secreted by 50,00o viable cells into the medium were calculated.

\section{Abbreviations}

$\mathrm{AR}$, androgen receptor; CK, cytokeratin; CV, coefficient of variation; EGFR, epidermal growth factor receptor; ELISA, enzyme-linked immunosorbent assay; EST, expressed sequence tag; $\mathrm{IC}_{50}$, half maximal inhibitory concentration; PDGFR, platelet-derived growth factor receptor; PSA, prostate-specific antigen (also known as kallikrein 3); SFK, Srcfamily kinase; TGF, transforming growth factor; uPA, urokinase-type plasminogen activator.

\section{Authors' contributions}

XDW designed the study, performed data analyses, and wrote the manuscript. KR performed the EphA2 Western blot analysis. FRL contributed data on the down-regulation of uPA protein in PC3 cells following drug treatment. LAX provided statistical assistance. FL shared insight and provided support for the study. EC helped conceive the study and edited the 
manuscript. FH helped conceive the study, advised on study design, and edited the manuscript.

\section{Additional data files}

The following additional data are available with the online version of this paper. Additional data file 1 is a table listing biomarkers correlated with sensitivity or resistance to dasatinib. Additional data file 2 is a table listing common predictive markers identified in prostate and breast preclinical models. Additional data file 3 provides microarray data on baseline gene expression of cell lines used for identification of genes whose expression correlated with in vitro sensitivity to dasatinib. Additional data file 4 provides microarray data on gene expression of cell lines treated with dasatinib or DMSO control.

\section{Acknowledgements}

We thank Shujian Wu and Mark Ayers for helpful discussions, and Shinta Cheng, Lewis Strauss, Maurizio Voi and Nicholas Dracopoli for critical reading of the manuscript.

\section{References}

I. Jemal A, Siegel R, Ward E, Murray T, Xu J, Smigal C, Thun MJ: Cancer statistics, 2006. CA Cancer I Clin 2006, 56:106-130.

2. Denmeade SR, Isaacs JT: A history of prostate cancer treatment. Nat Rev Cancer 2002, 2:389-396.

3. Tannock IF, de Wit R, Berry WR, Horti J. Pluzanska A, Chi KN, Oudard S, Theodore C, James ND, Turesson I, et al.: Docetaxel plus prednisone or mitoxantrone plus prednisone for advanced prostate cancer. N Engl J Med 2004, 35 I: I502-I5 I 2.

4. Pegram MD, Lipton A, Hayes DF, Weber BL, Baselga JM, Tripathy D, Baly D, Baughman SA, Twaddell T, Glaspy JA, et al.: Phase II study of receptor-enhanced chemosensitivity using recombinant humanized anti-p/85HER2/neu monoclonal antibody plus cisplatin in patients with HER2/neu-overexpressing metastatic breast cancer refractory to chemotherapy treatment. J Clin Oncol 1998, 16:2659-2671.

5. Castro M: The simpleton's error in drug development. J Clin Oncol 2002, 20:4606-4607.

6. Betensky RA, Louis DN, Cairncross JG: Influence of unrecognized molecular heterogeneity on randomized clinical trials. J Clin Oncol 2002, 20:2495-2499.

7. Lombardo LJ, Lee FY, Chen P, Norris D, Barrish JC, Behnia K, Castaneda S, Cornelius LA, Das J, Doweyko AM, et al.: Discovery of N(2-chloro-6-methyl-phenyl)-2-(6-(4-(2-hydroxyethyl)-piperazin- I-yl)-2-methylpyrimidin-4-ylamino) thiazole-5-carboxamide (BMS-354825), a dual Src/Abl kinase inhibitor with potent antitumor activity in preclinical assays. J Med Chem 2004, 47:6658-666I.

8. Huang F, Reeves K, Han X, Fairchild C, Platero S, Wong TW, Lee F, Shaw P, Clark E: Identification of candidate molecular markers predicting sensitivity in solid tumors to dasatinib: rationale for patient selection. Cancer Res 2007, 67:2226-2238.

9. Shah NP, Tran C, Lee FY, Chen P, Norris D, Sawyers CL: Overriding imatinib resistance with a novel ABL kinase inhibitor. Science 2004, 305:399-40I.

10. Talpaz M, Shah NP, Kantarjian H, Donato N, Nicoll J, Paquette R, Cortes J, O'Brien S, Nicaise C, Bleickardt E, et al: Dasatinib in imatinib-resistant Philadelphia chromosome-positive leukemias. N Engl J Med 2006, 354:253I-254I.

11. Yeatman TJ: A renaissance for SRC. Nat Rev Cancer 2004, 4:470-480.

12. Ishizawar R, Parsons SJ: c-Src and cooperating partners in human cancer. Cancer Cell 2004, 6:209-2I4.

13. Nam S, Kim D, Cheng JQ, Zhang S, Lee JH, Buettner R, Mirosevich J, Lee FY, Jove R: Action of the Src family kinase inhibitor, dasat- inib (BMS-354825), on human prostate cancer cells. Cancer Res 2005, 65:9185-9189.

14. Walker-Daniels J, Coffman K, Azimi M, Rhim JS, Bostwick DG, Snyder P, Kerns BJ, Waters DJ, Kinch MS: Overexpression of the EphA2 tyrosine kinase in prostate cancer. Prostate 1999, 41:275-280.

15. Bell SM, Brackenbury RW, Leslie ND, Degen JL: Plasminogen activator gene expression is induced by the src oncogene product and tumor promoters. J Biol Chem 1990, 265:1333-1338.

16. Singh D, Febbo PG, Ross K, Jackson DG, Manola J, Ladd C, Tamayo P, Renshaw AA, D'Amico AV, Richie JP, et al:: Gene expression correlates of clinical prostate cancer behavior. Cancer Cell 2002, I:203-209.

17. Marker PC, Donjacour AA, Dahiya R, Cunha GR: Hormonal, cellular, and molecular control of prostatic development. Dev Biol 2003, 253:165-174.

18. Litvinov IV, Vander Griend DJ, Xu Y, Antony L, Dalrymple SL, Isaacs JT: Low-calcium serum-free defined medium selects for growth of normal prostatic epithelial stem cells. Cancer Res 2006, 66:8598-8607.

19. Lin AM, Rini BI, Derynck MK, Weinberg V, Park M, Ryan CJ, Rosenberg JE, Bubley G, Small EJ: A phase I trial of docetaxel/estramustine/imatinib in patients with hormone-refractory prostate cancer. Clinical genitourinary cancer 2007, 5:323-328.

20. Lin AM, Rini BI, Weinberg V, Fong K, Ryan CJ, Rosenberg JE, Fong L, Small E): A phase II trial of imatinib mesylate in patients with biochemical relapse of prostate cancer after definitive local therapy. BJU international 2006, 98:763-769.

21. Guo L, Lobenhofer EK, Wang C, Shippy R, Harris SC, Zhang L, Mei $\mathrm{N}$, Chen T, Herman D, Goodsaid FM, et al:: Rat toxicogenomic study reveals analytical consistency across microarray platforms. Nat Biotechnol 2006, 24: I I62-II69.

22. Goldenberg-Furmanov M, Stein I, Pikarsky E, Rubin H, Kasem S, Wygoda M, Weinstein I, Reuveni H, Ben-Sasson SA: Lyn is a target gene for prostate cancer: sequence-based inhibition induces regression of human tumor xenografts. Cancer Res 2004, 64: 1058-1066.

23. Finn RS, Dering J, Ginther C, Wilson CA, Glaspy P, Tchekmedyian N, Slamon DJ: Dasatinib, an orally active small molecule inhibitor of both the src and abl kinases, selectively inhibits growth of basal-type/"triple-negative" breast cancer cell lines growing in vitro. Breast Cancer Res Treat 2007.

24. Charafe-Jauffret E, Ginestier C, Monville F, Finetti P, Adelaide J, Cervera N, Fekairi S, Xerri L, Jacquemier J, Birnbaum D, et al.: Gene expression profiling of breast cell lines identifies potential new basal markers. Oncogene 2006, 25:2273-2284.

25. Perou CM, Sorlie T, Eisen MB, van de Rijn M, Jeffrey SS, Rees CA, Pollack JR, Ross DT, Johnsen H, Akslen LA, et al.: Molecular portraits of human breast tumours. Nature 2000, 406:747-752.

26. Sorlie T, Tibshirani R, Parker J, Hastie T, Marron JS, Nobel A, Deng S, Johnsen H, Pesich R, Geisler S, et al.: Repeated observation of breast tumor subtypes in independent gene expression data sets. Proc Natl Acad Sci USA 2003, 100:84 I 8-8423.

27. Lapointe J, Li C, Higgins JP, van de Rijn M, Bair E, Montgomery K, Ferrari M, Egevad L, Rayford W, Bergerheim U, et al.: Gene expression profiling identifies clinically relevant subtypes of prostate cancer. Proc Natl Acad Sci USA 2004, I 1 I:81 I-816.

28. Abate-Shen C, Shen MM: Molecular genetics of prostate cancer. Genes Dev 2000, 14:2410-2434.

29. Patrawala L, Calhoun T, Schneider-Broussard R, Li H, Bhatia B, Tang S, Reilly JG, Chandra D, Zhou J, Claypool K, et al.: Highly purified CD44+ prostate cancer cells from xenograft human tumors are enriched in tumorigenic and metastatic progenitor cells. Oncogene 2006, 25:1696-1708.

30. van Bokhoven A, Varella-Garcia M, Korch C, Johannes WU, Smith EE, Miller HL, Nordeen SK, Miller GJ, Lucia MS: Molecular characterization of human prostate carcinoma cell lines. Prostate 2003, 57:205-225.

31. Chen CD, Welsbie DS, Tran C, Baek SH, Chen R, Vessella R, Rosenfeld MG, Sawyers CL: Molecular determinants of resistance to antiandrogen therapy. Nat Med 2004, 10:33-39.

32. Guo Z, Dai B, Jiang T, Xu K, Xie Y, Kim O, Nesheiwat I, Kong X, Melamed J, Handratta VD, et al.: Regulation of androgen receptor activity by tyrosine phosphorylation. Cancer Cell 2006, 10:309-319.

33. Cozzi PJ, Wang J, Delprado W, Madigan MC, Fairy S, Russell PJ, Li Y: Evaluation of urokinase plasminogen activator and its receptor in different grades of human prostate cancer. Hum Pathol 2006, 37:|442-|45|. 
34. Rocchi P, Muracciole X, Fina F, Mulholland DJ, Karsenty G, Palmari J, Ouafik L, Bladou F, Martin PM: Molecular analysis integrating different pathways associated with androgen-independent progression in LuCaP 23.I xenograft. Oncogene 2004, 23:9111-9119.

35. Pulukuri SM, Gondi CS, Lakka SS, Jutla A, Estes N, Gujrati M, Rao JS: RNA interference-directed knockdown of urokinase plasminogen activator and urokinase plasminogen activator receptor inhibits prostate cancer cell invasion, survival, and tumorigenicity in vivo. J Biol Chem 2005, 280:36529-36540.

36. Miyake H, Hara I, Yamanaka K, Arakawa S, Kamidono S: Elevation of urokinase-type plasminogen activator and its receptor densities as new predictors of disease progression and prognosis in men with prostate cancer. Int J Oncol I999, I 4:535-54I.

37. Shariat SF, Roehrborn CG, McConnell JD, Park S, Alam N, Wheeler TM, Slawin KM: Association of the circulating levels of the urokinase system of plasminogen activation with the presence of prostate cancer and invasion, progression, and metastasis. J Clin Oncol 2007, 25:349-355.

38. Urban P, Vuaroqueaux V, Labuhn M, Delorenzi M, Wirapati P, Wight E, Senn HJ, Benz C, Eppenberger U, Eppenberger-Castori S: Increased expression of urokinase-type plasminogen activator mRNA determines adverse prognosis in ErbB2-positive primary breast cancer. J Clin Oncol 2006, 24:4245-4253.

39. Janicke F, Prechtl A, Thomssen C, Harbeck N, Meisner C, Untch M, Sweep CG, Selbmann HK, Graeff H, Schmitt M: Randomized adjuvant chemotherapy trial in high-risk, lymph node-negative breast cancer patients identified by urokinase-type plasminogen activator and plasminogen activator inhibitor type I. J Natl Cancer Inst 200 I, 93:913-920.

40. Thomas SM, Brugge JS: Cellular functions regulated by Src family kinases. Annu Rev Cell Dev Biol 1997, I3:51 3-609.

4I. Eisen MB, Spellman PT, Brown PO, Botstein D: Cluster analysis and display of genome-wide expression patterns. Proc Natl Acad Sci USA 1998, 95:|4863-| 4868. 